\title{
THE HEART ' RHYTHM IN DIPHTHERIA
}

\section{CARL SCH WENSEN}

From Blegdams-Hospital, Copenhagen

For the last 25 years of the past century clinicians have agreed on the serious significance of the symptoms of cardiac weakness during an attack of diphtheria, and numerous are the publications on this question. All authors hold that the chief features are: murmurs, dilatation and irregularities of the rhythm of the heart. Unfortunately, they have not been able to record the latter graphically, but several, such as Dubrisay, ${ }^{1}$ and Huguenin, ${ }^{2}$ describe this abnormal rhythm distinctly, in fact so distinctly that we are able to classify many of the disturbances. After the study of the rhythm of the heart gained ground in the ordinary clinical examinations, the following cases were published with sufficient graphic evidence.

Cases of acute heart-block by: Bøe, ${ }^{3}$ Fleming and Kennedy, ${ }^{4}$ Hecht, ${ }^{5}$ Hume, and Rohmer." A most interesting case of acute heart-block combined with auricular fibrillation is reported by $\mathrm{J}$. Parkinson. ${ }^{8}$ His patient first developed complete heartblock; 4 days later auricular fibrillation set in. The fibrillation was, as shown by the electrocardiograph, still present 6 months after its onset. This is the only case of auricular fibrillation during diphtheria recorded with definite evidence. In a case recorded by Price and Ivy Mackenzie? the tracings are not convincing.

Cases of auricular flutter during diphtheria are reported by Hume," who proposes that the tracings should be of "nodal-rhythm" with attacks of flutter.

A case of paroxysmal tachycardia is reported also by Hume." The patient had had extrasystoles from the 15th day of his illness.

Records are published by Aviragnet, Lutembacher et le Soulier ${ }^{10}$ and Aviragnet et Lutembacher ${ }^{11}$ who declare them to be the result of extrasystoles and heart-block; but the tracings are not convincing.

Received for publication, August 29, 1921.

1 Union med., 1877, p. 288.

Rev, de med., 1888, pp. 790 and 995 .

- Med. Rec., 1913, 30, p. 73.

- Heart, 1910, 2, p. 77.

s Erg. d. inn. Med. u. Kinderh., 1913, 11, p. 324.

- Heart, 1913, 5, p. 25.

7 Ztschr. f. exper. Path. u. Therap., 1912, 11, p. 426.

8 Heart, 1918, 6, p. 13.

- Ibid., 1911, 3, p. 233.

10 Arch. d. mal. d. Coeur., 1918, 11, p. $41 ; 1920,13$, p. 1.

1 Jour. d. med. Fr., 1920. 


\section{METHODS OF INVESTIGATION}

For about 4 years I have had the opportunity of following several thousands of patients with diphtheria during their stay in the hospital. As the toxicity of diphtheria vares in different epidemics I have thought it best to consider only patients seen during a limited space of time, and I have chosen patients who were discharged or who died between Jan. 1 and July 1, 1918. As cardiac disturbances usually occur only in patients with grave cases of diphtheria, I determined to follow these specially and to follow the lighter cases only when the patients had shown symptoms of cardiac trouble. By "grave" diphtheria is understool diphtheria with membrane on both the tonsils, more or less of the uvula and the soft palate. In several of the cases it even reached the gums. Furthermore, there was a more or less pronounced periadenitis of the glands of the neck.

In all, 568 patients suffering from diphtheria passed through the hospital during the period mentioned; 118 had cases of grave diphtheria. These patients were followed throughout their stay in hospital. From the day of admittance they were examined 2 or 3 times weekly. In the gravest cases, in which I could expect a quick development of the symptoms, I examined the patients daily, ofter several times daily. The pulse was taken morning and evening. The examinations have been: ordinary stethoscopic examination, graphic recording if signs of disordered action of the heart was found, and determination of the blood pressure.

Stcthoscopic Findings.-Dilatation of the heart was found in 60 cases, in 42 patients the dilatation involved both the right and left half, in 11 only the right and in 7 only the left half.

We often saw systolic murmurs develop during the stay in hospital; they were usually soft, but might be very harsh; a general feature of the murmurs was that they often changed from day to day. Besides the murmurs, another change of the suunds was found: they became accentuated; then they were muffled and faint; at times they became quite inaudible. The last was a bad prognostic sign and was only found at the first sound in very serious cases. These alterations may vary much from day to day, even from hour to hour. Ordinary systolic murmurs were found in 88 cases; in 74 cases the murmurs were both apical and pulmonal; in 9 cases the murmur was only apical, and in 5 only pulmonal.

Disturbances in Rate and Rhythm.-The rate usually fell with the temperature and was low for a fortnight, whereafter it rose again. 
Often it was constantly low in the beginning, morning and evening alike; later came a jumping of the rate, a difference of about 20 not being uncommon. The rate was then always highest in the evening. In several of these ordinary cases of bradycardia during convalescence injection of atropine gave a pronounced rise in the pulse rate.

During the daily work with the patients it soon became evident that 2 quite distinctly different types of irregularities occur besides the ordinary respiratory irregularity; the latter was often found even in patients who at the same time or later on in the course of the illness showed undeniable signs of impairment of the heart. I therefore find it quite impossible to consider this irregularity as a proof that the heart has escaped damage, as Mackenzie does.

The two types of disturbed rhythm were: (a) One which occurred early in the disease, usually before the membrane had entirely disappeared. These patients all died with symptoms of heart failure in the course of a few hours or days after the abnortual rhythm was found. Of the 118 grave cases, 5 were fatal in the acute stage of the disease; all died with symptoms of heart-failure; in 3 of these this abnormal ryhthm was found. (b) One which appeared late in the disease consisted of extrasystoles. In some cases they were found only at one examination, but usually they were present for several weeks until at last they disappeared. None of these patients died with symptoms of heartfailure.

When the abnormal rhythm started in the early stage of the illness (in my cases latest on the 11 th day), it was always quite impossible to determine the type by simple comparison of the pulse and the auscultation; in these cases I have taken polygraphic tracings, as I unfortunately had no opportunity of using the electrocardiograph. I have also taken tracings from several of the patients with extrasystoles with the object of verifying the diagnosis, but all authors agree that it is possible to recognize this irregularity of the pulse by simultaneous comparison between the pulse and the auscultation, and these abnormal contractions often occurred with such long intervals that it would have been practically impossible to catch them. Furthermore, I found several times that they had disappeared during the application of the polygraph; as the children became anxious their pulse-rate rose, and it is well known that the premature contractions disappear when the rate of the normal irritations from the sinus is high enough to prevent the heterotope irritation from producing a contraction of the heart-muscle. For these reasons I ceased using the polygraph at this stage of the diphtheria. 


\section{A. THE "EARLY" TYPE}

In the limited space of time between $1 / 1-1_{v} / 7,1918$, I only had the opportunity of examining 3 cases displaying an early appearing irregularity, but as I later on have examined 8 , I have in all examined 11 cases of this kind.

The age varied from $1 \frac{1}{4}$ to $91 / 2$ years; 6 of the patients were boys, 5 girls. All 11 died. Death occurred at the earliest on the 7 th day of illness, at the latest on the 13th day, on an average on the 10th day. The irregularity was earliest found on the 6 th day, latest on the 11 th, on an average on the 8 th day of the diphtheria

The time that passed between the appearance of the irregularity and death was as follows: One patient died on the same day as the irregularity appeared; 5 patients died the day after; two died 2 , one 3 ,

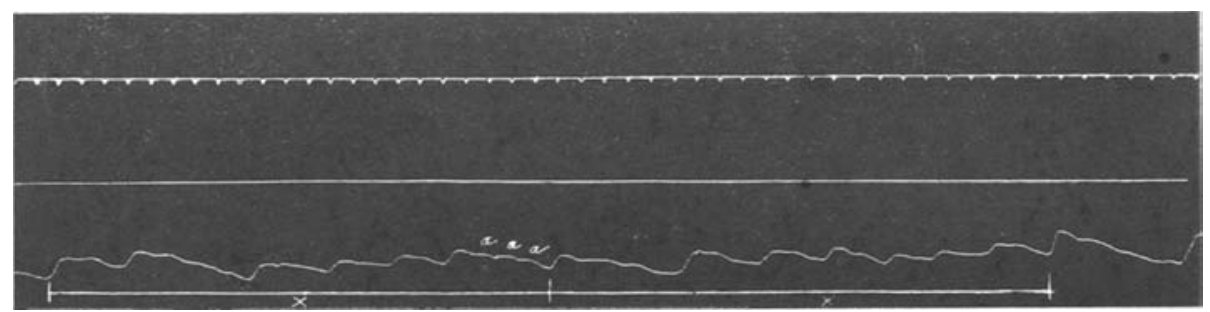

Chart 1, Case 1.-The tracings showed a regular pulse for 13 beats, then the rate of the radial pulse became as shown in the chart. There is pronounced spacing. These tracings were taken with a Jacquet's polygraph. The time-marking always corresponds to $1 / 5^{\prime \prime}$. The upper curve is always the jugular, the lower the radial.

one 4 and one 6 days after the irregularity had appeared. In 10 of the 11 cases I found, besides the irregularity, ordinary symptoms of heartfailure (dilatations, murmurs); in 1 case the irregularity was the sole symptom (case 11). As mentioned before, I took polygraphic tracings in all but one case in which the patient was $11 / 4$ year old. None of the patients were under treatment with digitalis or similar drugs.

In 5 of the cases $I$ made the atropine-test with negative results (cases 2, 5, 6, 7 and 9).

In 4 cases (Nos. 1, 2, 4 and 5) I examined the bundle of HisTawara histologically according to Tawara. ${ }^{12}$ I found it the seat of pathologic changes to the same degree as the myocardium surrounding it; most pronounced was a diffuse cellular infiltration. In none of the cases was an interruption of the bundle found.

12 Reiszleitung-syst. d. Säugetiereherzen, 1906, p. 37. 
I shall give a short summary of the cases with regard to the abnormalities in rate and rlyythm, together with the tracings obtained.

\section{Summary of Cases}

CASE 1.-A 4 year old girl, admitted on the 4 th day, died on the 12th day of illness. On the 11 th day the pulse rate fell from about 100 to 62 , it was alternately regular (fig. 1).

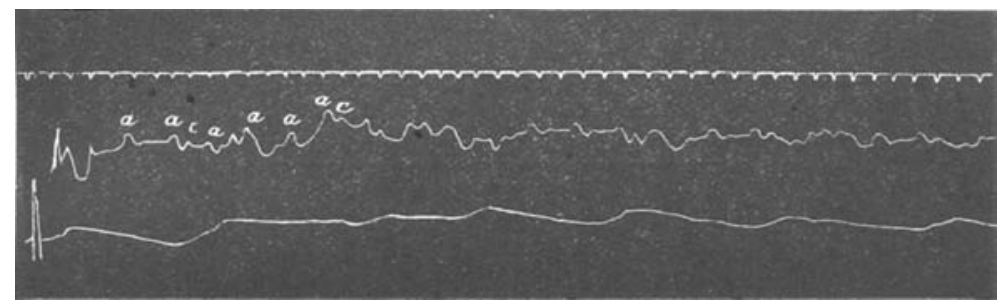

Chart 2, Case 2.-The tracings show partial heartblock. The auricular rate is about 120 per minute, the ventricular rate only about 50 .

CASE 2.-A 3 year old boy, admitted on the 5th day, died on the 13th day of illness. The pulse rate fell during the night between the 9 th and the 10 th day from 120 to 68 , and the pulse became irregular. Tracings (fig. 2) showed partial heartblock. The atropine test gave negative results. The tracings remained unaltered until death.

CASE 3.-An 8 year old girl, admitted on the 3rd day, died on the 12th day of illness. During the night between the 5 th and 6th day the pulse rate fell from 100 to 48 ; the tracings showed partial heartblock (fig. 3). On the 7th

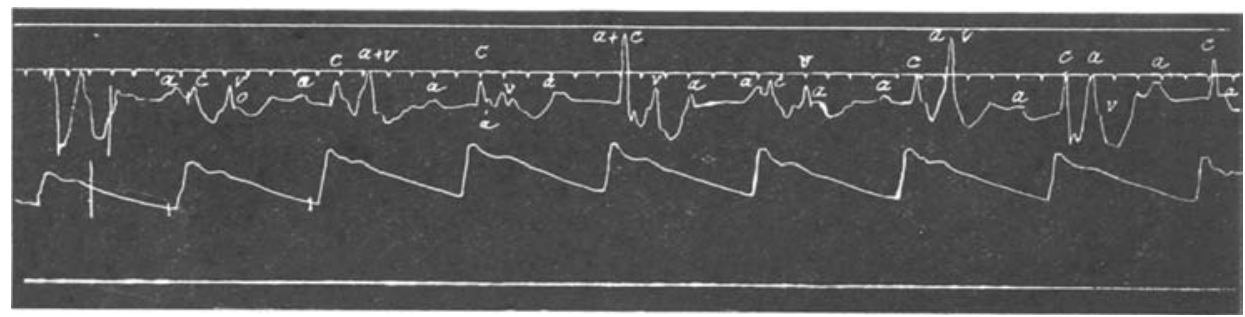
larity.

Chart 3, Case 3.-Typical partial heartblock. The a-c interval varies with great regu-

and the 8th day the tracings still showed varying degrees of partial heartblock, but on the 911 day the pulse had become more irregular and changing. After I had obtained 3 tracings like fig. 4 the pulse suddenly changed and became fast and regular (120 per minute) as in fig. 5 . During 20 minutes the pulse remained unaltered. but thereafter the tracings again became like those in fig. 4. The last days the patient lived the pulse showed features like those on the 9th day (figs. 6 and 7 ). 


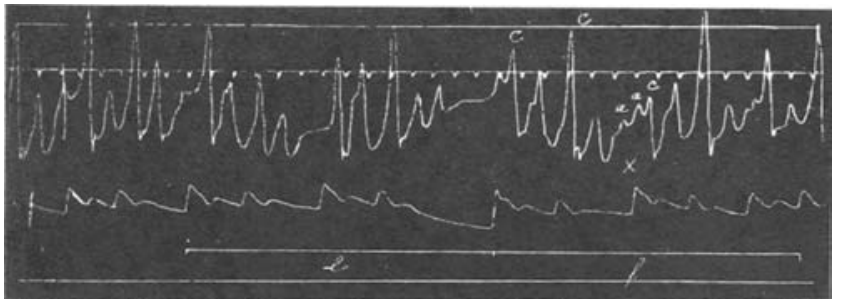

Chart 4, Case 3.- The radial curve is very irregular, but there is pronounced spacing. There are great, rather uniform waves in the jugular curve. At " $x$ " small waves are seen marked "a," which may signify auricular contractions.

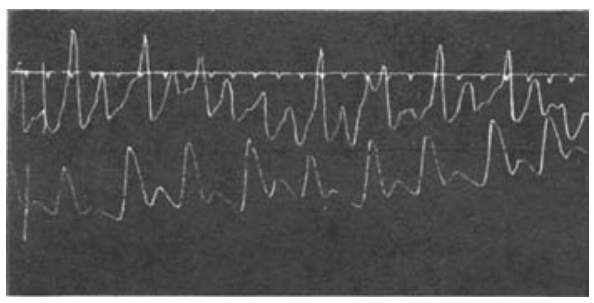

Chart 5, Case 3.-Tachycardia with a rate of about 120 . The jugular curve is of the ventricular form.

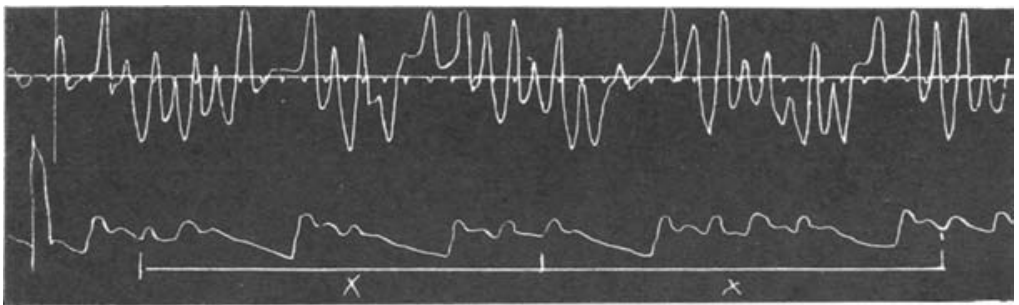

Chart 6, Case 3.-There are uniform big waves in the jugular curve. The radial curve shows pronounced spacing.

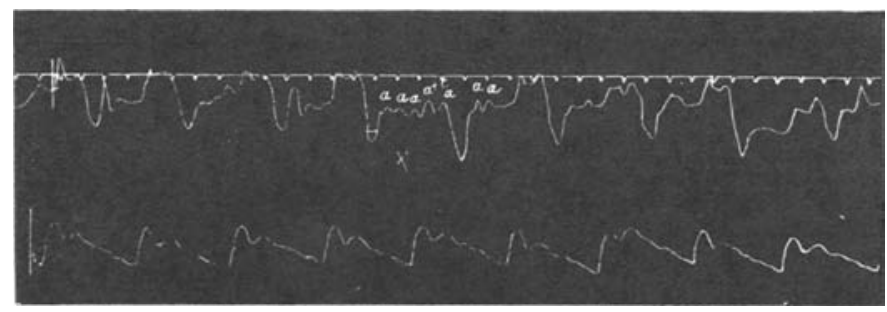

Chart 7, Case 3,-The radial curve is now regular. At " $x$ " in the jugular tracing small waves marked "a" are seen. 
CASE 4.-A 5 year old boy admitted on the 3 rd day died on the 8 th day of illness. On the 6 th day the pulse became irregular (fig. 8) about 80 . The same day as the patient died the pulse was about 180 , regular.

CASE 5.-An 8 year old girl admitted on the 4th day died on the 9th day of illness. On the 6th day the tracings showed partial heartblock (fig. 9); the atropine-test gave negative results. On the 7 th day consecutive tracings were like those of fig. 10 and 11, the pulse varying highly. On the 8th day tracings were like fig. 11.

CASE 6.-An 8 year old boy admitted on the 4th day died on the 13th day of illness. On the 12th day the pulse rate had fallen to between 40 and 50 . Tracings showed partial heartblock (fig. 12). The atropine-test gave negative results. On the 13th day the pulse varied much in rate and rhythm, in the morning it was 40 , in the forenoon 140 . Later it was 72 and irregular, and while I observed it, it suddenly changed to 112 , regular (fig. 13).

CASE 7.-An 8 year old boy admitted on the 3 rd day, died on the 8 th day of illness. On the 7 th day the pulse became irregular but no tracings were obtained. On the 8 th day the rate in the morning was 120 , later it fell to

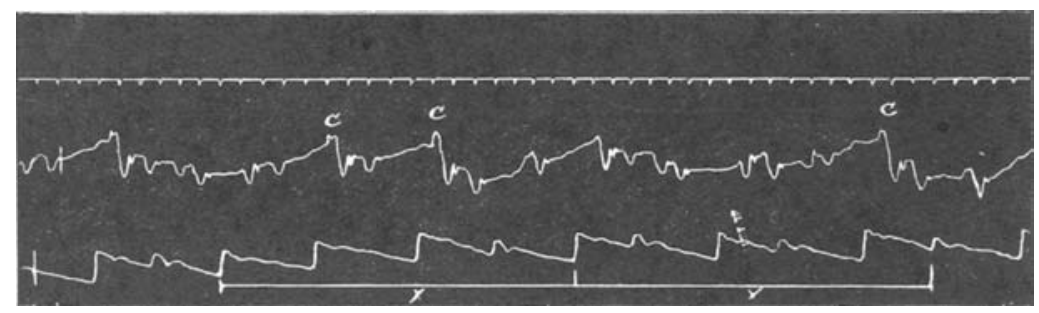

Chart 8 , Case 4.-The radial curve is irregular but with spacing.

between 60 and 64 , and the pulse was quite regular. At 8 o'clock in the evening a tracing was obtained which showed irregularity as in fig. 14 . The atropine-test gave negative results; the tracings remained as before. Half an hour later he suddenly was seized by convulsions and died in a minute.

CASE 8.-A $91 / 2$ year old boy almitted on the 3rd day died on the 12 th day of illness. The rate was about 60 on the 9 th day, rose to 120 on the 11 th day, and the pulse became irregular. Tracings obtained were like those in fig. 15. The tracings obtained on the following day were unaltered. He died during a fit of convulsions.

CASE 9.-A $2^{1 / 2}$ year old girl admitted on the 3rd day died on the 7 th day of illness. In the course of the night between the 4th and the 5th day the pulse rate had fallen from 100 to 68 , the rhythm was quite regular. On the 6 th day the rate was 68 , but the rhythm was now irregular (fig. 16). The atropine-test gave negative results. On the 7 th day the pulse changed much in rhythm (sometimes it was slow, sometimes fast), the rate being about 96; later the rate increased to 132 , and the rhythm was nearly regular, with only an occasional speeding up of the beat. She died in the course of the afternoon.

CASE 10.-A 7 year old boy admitted on the 5th day, died on the 11th day of illness. In the course of the 10th day the pulse rate fell from 112 in the morning to 66-68 in the middle of the day. Tracings showed features like those in fig. 17. After I had obtained one tracing the rhythm changed and 
the rate increased to 112-120. Tracings were like those in fig. 18 . On the 11th day the pulse varied much in rate and rhythm; tracings were like those in figs. 17 and 18 except that occasionally there was a longer pause in the radial pulse.

CASE 11:-A 15 month old girl admitted on the 4th day died on the 7 th day of illness. On the 7 th day the pulse became irregular with dropped beats (determined by auscultation) and the child died in the evening. No tracings were obtained.

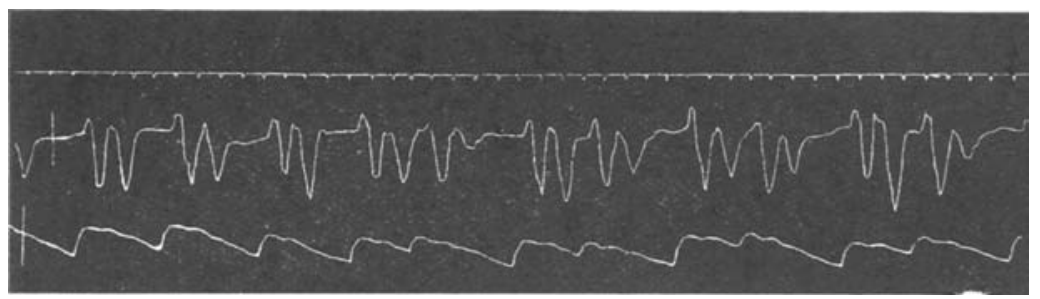

Chart 9, Case 5.-The tracings probably show partial heartblock. The atropine test gave negative results, the curves remaining unaltered.

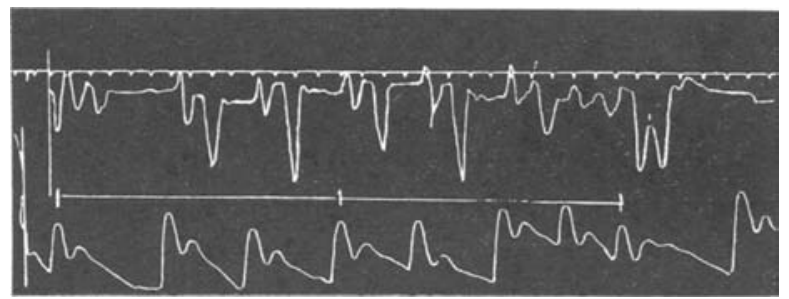

Chart 10, Case 5.-The radial pulse is irregular with pronounced spacing.

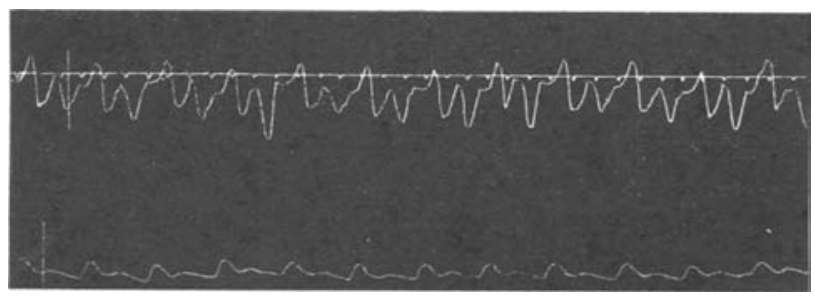

Chart 11. Case 5.-Regular tachycardia, the rate being about 90 . The venous curve is of the ventricular form.

As mentioned, only the electrocardiograph would have determined definitely the type of irregularities found in these cases, but unfortunately $I$ have not had the opportunity of using one. On this account I have not deemed it correct to take each tracing alone, but I have reached 
the following conclusions by considering both the tracings and the ordinary clinical symptoms and, last but not least, by comparing the findings from the different patients:

In the fatal cases of diphtheria, bradycardia often occurs at the end of the 1st or at the beginning of the 2nd week of illness. In some patients there was in a few hours a sudden drop in the pulse rate to about one half of the original rate.

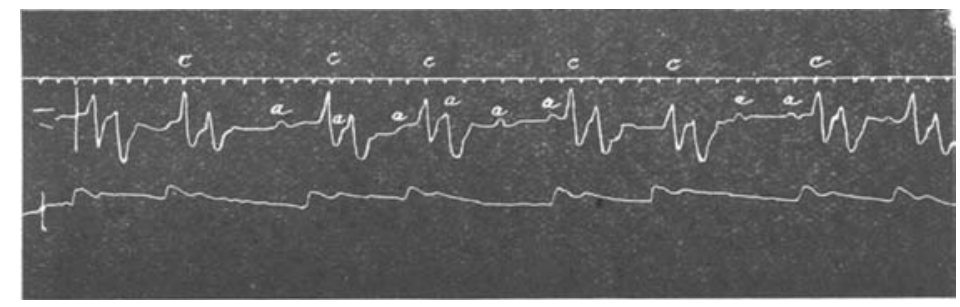

Chart 12, Case 6.-Typical partial heartblock.

The bradycardia was usually irregular, at times it was bigeminate; by auscultating, it was easy to distinguish between these bigeminate periods and extrasystoles.

In 4 of the 10 cases I obtained tracings in this period of bradycardia, and they all showed partial heartblock. In 3 of these cases the atropine-test gave negative results, the block, therefore, must have been caused by a direct affection of the bundle and could not have been due to vagal irritation.

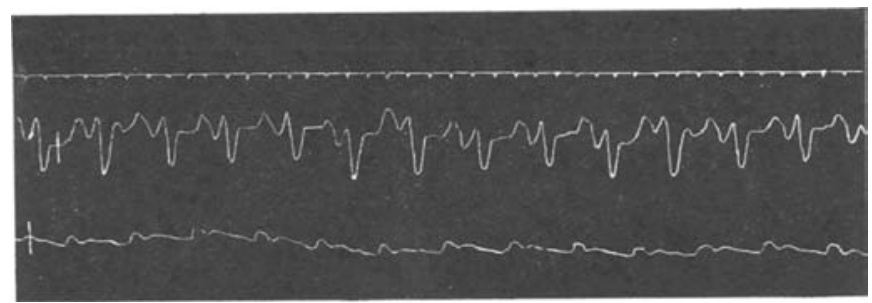

Chart 13, Case 6.- Regular tachycardia. The jugular curve is of the ventricular form.

In one of the cases the block persisted unaltered until death occurred on the 3rd day after the block had set in. In the 3 other cases the pulse later changed in rate and rhythm; at times the pulse rate was slow and regular, at others, bigeminate; occasionally there were series of quicker beats followed by a longer pause. Sometimes a regular tachycardia suddenly set in, followed by one of the other types. Auscultation 
showed that this varying irregularity did not in the least resemble extrasystoles. Soon the pulse rate became complex, and its most conspicuous character was the great instability of rhythm.

In the 6 cases in which I did not obtain tracings in the bradycardial period, the tracings obtained showed features just like those in tracings

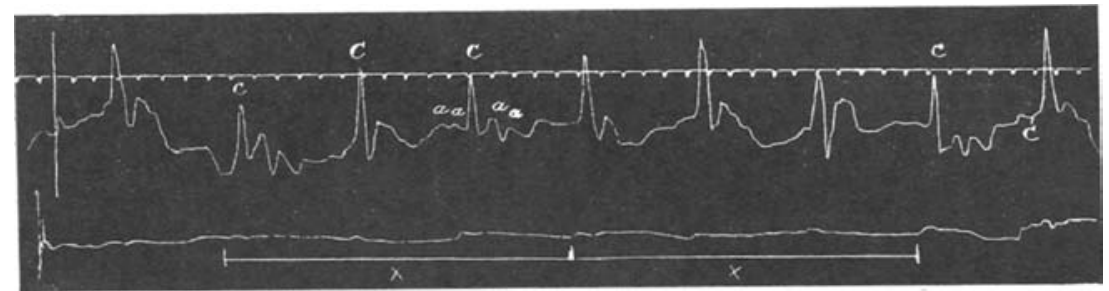

Chart 14, Case 7.-The radial tracing is irregular with spacing. In the jugular curve some small waves marked " $a$ " are seen; probably they are due to very quick auricular con. tractions.

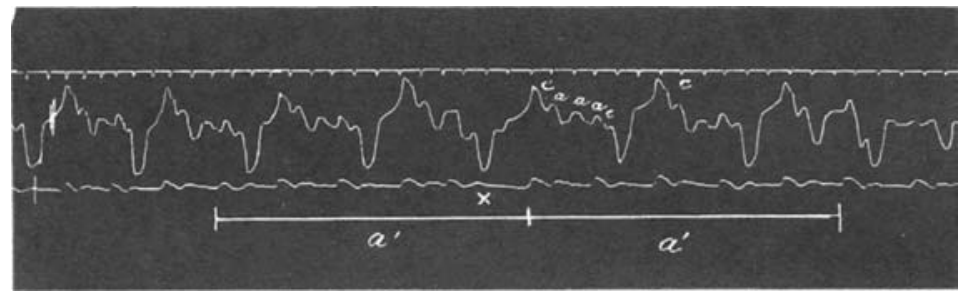

Chart 15, Case 8.-The radial curve shows spacing. The small waves marked "a" may correspond to auricular contractions.

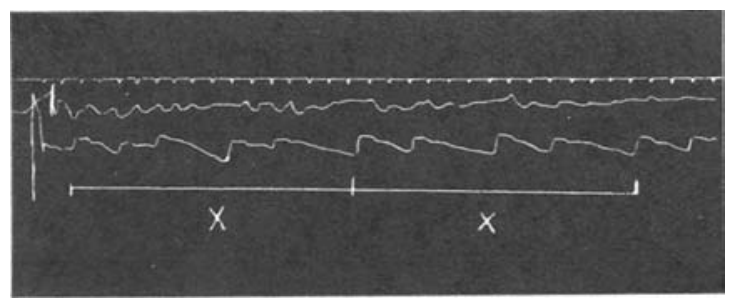

Chart 16, Case 9.-The radial tracing shows irregularity with spacing.

made during the complex period mentioned in the foregoing. In 2 of these cases the atropine test also gave a negative result.

With regard to the tracings from the complex period, I think it evident that they show the same type of irregularity in all of the 9 cases. Clinically there was no difference between these cases, and it was easy 
to recognize the irregularity by making the usual comparison between pulse and auscultation.

The most remarkable features of this disturbance of the rhythm are: (1) the instability of the rate and the rhythm, (2) the great waves which are found in several of the jugular tracings, (3) the pronounced "spacing" (Lewis ${ }^{13}$ ) shown by all these radial tracings, (4) smaller waves marked a in some of the jugular tracings may correspond to fast auricular contractions, and (5) the fact that the tracings are identical with tracings made in cases of flutter verified electrocardiographically (Lewis, ${ }^{14}$ Ritchie ${ }^{15}$ ).
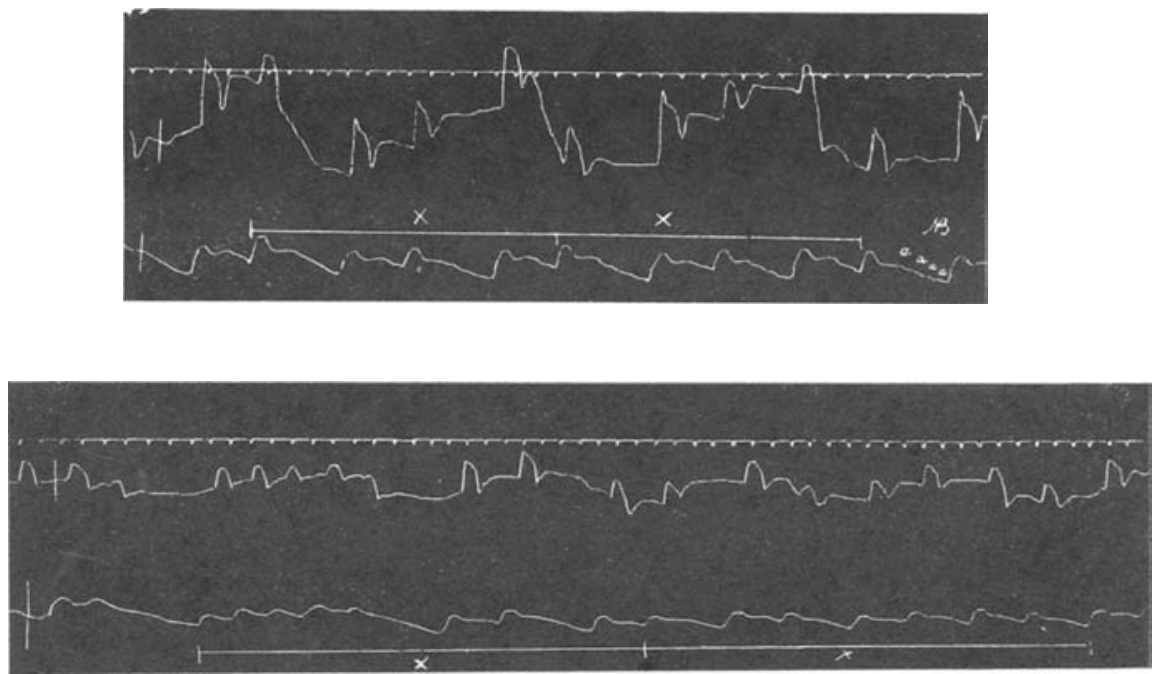

Chart 17 and 18, Case 10.-The radial curves show spacing.

Only the electrocardiograph could give a definite determination of this complex type. I think that the facts mentioned, however, indicate that the probable cause of this complex irregularity is that the original acute partial heart-block has been complicated with attacks of auricular flutter.

\section{B. THE "LATE" TYPE}

As I have mentioned already, extrasystoles were a usual finding in the convalescence after diphtheria. Five hundred and sixty-three patients with diphtheria have been examined with regard to the

13 Mech. of Graph. Registration of Hcart, 1920, p. 272.

14 Clin. Disorders of Heartbeat, 1913.

t5 Auricular Flutter, 1914. 
occurrence of extrasystoles; 113 of them had cases of grave diphtheria. Of all the 563 cases, $14 \%$ had extrasystoles during convalescence; in $61 \%$ of the 113 grave cases extrasystoles appeared. In all, 77 patients showed extrasystoles; 6 of them were adults, 71 less than 15 years old. The extrasystoles appeared at the earliest on the 18 th day, at the latest on the 55th day, on an average on the 33rd day. Nine patients had extrasystoles during the 3rd week, 6 during the 4th, 32 during the 5th, 15 during the first half of the 6th, 2 during the second half of the 6th, 10 during the 7th, and 3 during the 8th week of illness. In 19 cases the extrasystoles was the first abnormal symptom from the side of the heart and in 9 of these cases it was the only one.

As mentioned, extrasystoles were diagnosed by simultaneous comparison between the pulse and the action of the heart as found by auscultation. Usually they were found for several days or weeks and

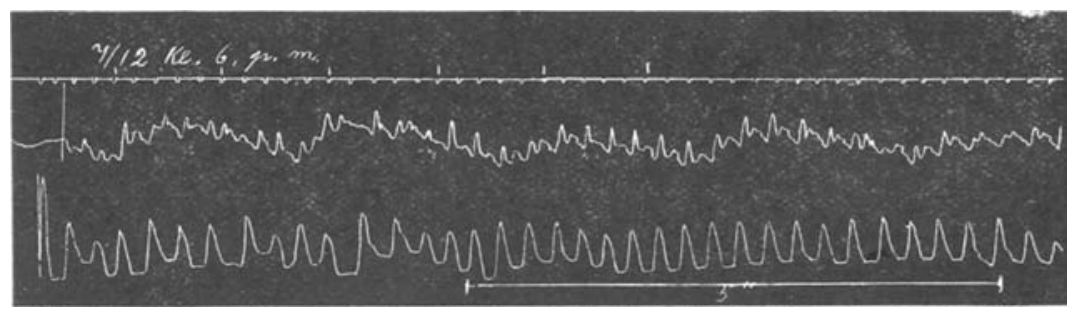

Chart 19.-The radial curve shows a regular tachycardia of about 236 per minute.

were very frequent (10 or more per 100 not uncommon); in some patients they occurred only with long intervals and during a few days; they were seldom found at only one examination. In not a few cases were found series of extrasystoles; in one case even paroxysmal tachycardia was found.

In the tracings $I$ took in these cases I found auricular as well as ventricular extrasystoles, but as I did not take tracings in all the cases for the reasons mentioned, I cannot pass any opinion as to which is the most common type: the auricular or the ventricular, and whether they always are of the same origin in the same patient.

As an example I give a short summary of the case in which the patient had attacks of paroxysmal tachycardia:

In a girl, aged 11 years, admitted on the 5th day and discharged on the 68 th day of illness, the tonsils, uvula and part of the soft palate were covered with membrane. There was some periadenitis. On the 18th day of illness the membrane had disappeared. On the 20 th day a soft systolic murmur was 
heard at the apex. On the 30th day there were extrasystoles with long intervals. The next day she was very nervous and cried. The pulse rate was, counted by auscultation, more than 200 per minute; the action was tumultuous. While I was preparing to make the tracings she stopped crying and the tracings showed normal conditions. At $6 . \mathrm{p}$. m. I obtained tracings like those shown in fig. 19 . The rate, counted on the tracing, was 236 per minute. Pressure of the vagus gave no retardation. At $6: 30 \mathrm{p}$. m. the character of the tracings had changed and showed a regular pulse of $1+4$ per minute, an occasional auricular extrasystole. The day after the pulse was 140 , the tracings showed extrasystoles of ventricular origin. The following day the tracings for some time were identical with those in fig. 19; then a series of extrasystoles of ventricular origin appeared (fig. 20). She had no more attacks of tachycardia, but for a fortnight she had extrasystoles. The patient was discharged without any symptom of disease of the heart.

Later I had the opportunity of examining with the electrocardiograph 31 patients who had dilatations, systolic murmurs and frequent extrasystoles during the 5 th week of their illness. The electrocardio-

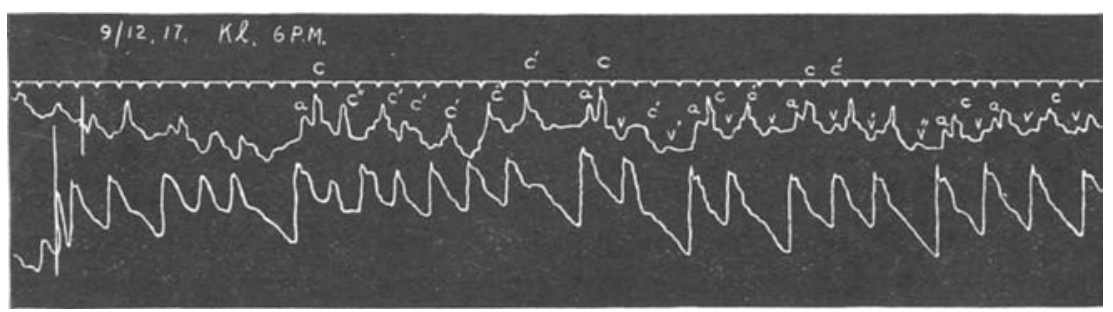

Chart 20 .-There are here a series of extrasystoles of ventricular origin.

grams were taken in the ordinary way from all 3 leads. In only 3 of the patients (children) I happened to catch the extrasystoles. In 2 cases they were ordinary auricular extrasystoles while in the 3rd case there was an extrasystole arising in the junctional tissues. The electrocardiograms of the other patients were quite normal.

DETERMINATION OF THE BLOOD PRESSURE

In 4 of the fatal cases and in 69 of the grave cases I repeatedly determined the blood pressure by Riva-Rocci's apparatus with a broad cuff.

In the 4 fatal cases the systolic blood pressure fell to $60,75,45$ and 53 , respectively. In the greater part of the 69 cases I found that the systolic blood pressure decreased slowly. The minimum was reached during the 2nd or 3rd week, whereafter the pressure again rose slowly until normal heights were reached during the 4 th or the 5 th week. In only 3 of these cases did the pressure fall below 70. A blood pressure 
below 70 must therefore be considered a bad prognostic sign. These finclings are in accordance with those of E. Faber. ${ }^{18}$

\section{DISCUSSION}

During the course of diphtheria, murmurs, dilatation and irregularity are according to my experience a usual phenomenon. These symptoms may be assumed to be the result of an acute inyocarditis; the murmurs indicate not a valvulitis, but an inefficiency of the functions of the muscles around the mitral valve and of the papillary muscle (Mackenzie, ${ }^{17} \mathrm{Krehl}{ }^{18}$ ). The great instability of the nurmurs indicates that they are not caused by a valvulitis; furthermore, valvulitis is never discovered postmortem.

In 98 , or $17 \%$ of my 568 cases and in 89 , or $75 \%$, of the cases of grave diphtheria, these symptoms of myocardial disease were observed.

Of these 98 cases, 60 patients had both murmurs and dilatation, 28 only murmurs but no dilatation; in 1 case the early appearing irregularity and in 9 extrasystoles were the sole heart symptoms showing clisturbances of that organ.

Of the 84 patients with a grave diphtheria who survived the acute stage of illness and in whom dilatation and murmur or murmur alone were found, extrasystoles occurred later in 68 , or in $81 \%$ of the cases.

The first symptom of myocarditis was found earliest on the 3rd day of illness, latest (as extrasystoles) on the 47th day, and on an average on the 15th day of illness. If we do not consider the patients in whom the extrasystoles were the first abnormal sign, the usual time for the appearance of the first symptom of myocardits was on the 10th day of illness.

The question is whether this acute myocardits explains all the clinical symptoms found during diphtheria or whether it will be necessary to assume vasomotor paresis or nervous influences.

It is a well-known fact that in diphtheria more than in any other acute disease there is a tendency to fatal syncope. In the cases in which death occurred in the acute stage (2nd or 3rd week) there were always found symptoms of a severe myocarditis (dilatation and murmurs). This in itself can explain the fatal course but cannot explain the frequent attacks of syncope, but when this grave acute myocarditis.

10 Dödsaarsagene ved Difteri, 1903.

17 Diseases of Heart, 1914, p. 304.

18 Erkrank. d. Herimuskels, etc., 1913, p. 373. 
as I have proved, often is combined with a great disturbance of the rhythm ${ }^{10}$ the matter is greatly simplified.

This early irregularity could, as a rule, be divided into 2 periods : in the first only varying degrees of heartblock were found, in the second was found the irregularity which $I$ have described as the complex irregularity.

The period of heartblock makes it clear why these patients are prone to attacks of syncope and sudden death; as the result of profound slowing or prolonged standstill of the ventricle these patients have attacks of cerebral anemia.

As to the complex irregularity, Mackenzie ${ }^{17}$ and Lewis ${ }^{14}$ say that severe disturbance ${ }^{20}$ of the rhythm of the heart often produces attacks of syncope as the result of cerebral anemia, especially when the muscle is damaged beforehand. Furthermore, Wenckebach ${ }^{21}$ shows how the simultaneous contractions of the auricles and the ventricles are a hindrance in the emptying of the auricles; the blood accumulates in the central veins and in the liver; therefore there is a diminished output of the left ventricle. When the rhythm varies greatly, this abnormal mechanism may produce attacks of cerebral anemia with syncope. I have considered the consequences of the complex irregularity only as irregularity, but if these patients present cases of flutter, the attacks of syncope are only such as one usually finds in cases of flutter, in which the ventricle occasionally answers to the full call of the auricle and the heart rate leaps to its fullest. Then the blood pressure sinks rapidly, and we get syncope as a result of cerebral anemia. If these seizures are of prolonged duration, death intervenes (Lewis ${ }^{20}$ ).

Among the other important symptoms in this stage of the diphtheria the following are the most significant: enlargement of the liver, low blood pressure with coolness and pallor of the skin, vomiting, and the absence of symptoms of pulmonary stasis.

The lesser degrees of enlargement of the liver, as well as of the decrease in blood pressure, can be explained as the result of a failing heart (Sutherland ${ }^{22}$ ). In the fatal cases we often see an excessive enlargement, which appears in the course of a few hours; the liver often reaches below the umbilical-line and is very tender. This excessive enlargement can be explained by the severe irregularity of the rhythm

\footnotetext{
18 Between Jan. 1 and Jan. 7, 1918, this abnormal rhythm was observed in 3 of the 5 patients who died in the acute stage.

20 Lectures on the Heart, 1914.

21 Die unregelm. Herztätigkeit, 1914.

as The Heart in Early Lifc, 1914.
} 
of the heart (Mackenzie, ${ }^{17}$ Wenckebach ${ }^{21}$ ), just as we often find the liver greatly enlarged in patients with paroxysmal tachycardia, especially when the heart muscle is damaged.

The low blood pressure, together with the coolness and pallor of the skin which are very serious signs in diphtheria, are a usual finding in cardial syncope and can be explained by the diminished output of the left ventricle, the blood being accumulated in the central veins and in the liver. The vomiting is a result of the cerebral anemia.

That we in these patients do not find pronounced signs of pulmonary stasis can be explained by the fact that blood is accumulated in the central veins and in the liver. For this reason the right ventricle gets very little blood, and is accordingly unable to produce a pulmonary stasis (Wenckebach ${ }^{21}$ ).

The early appearing abnormal rhythm (heartblock or "complex irregularity") which I have found in diphtheria, explains why patients die without the pathologist being able to find a sufficient cause for death; and as this abnormal rhythm is easily overlooked-if one does not specially look for it and if one examines the pulse only occasionally - it is evident that in former days there has been uncertainty as to the cause of death and that other explanations than the impairment of the heart have been sought.

For this reason the theory of vasomotor paresis or paresis of the vagal nerve has been put forward. Against this theory is the fact that instead of the increase of the pulse rate which was to be expected, we not only have bradycardia in the fatal cases with heartblock, but as mentioned before, there is in most cases a greater or smaller decrease of the rate, often a veritable bradycardia (Romberg, ${ }^{23}$ Schmaltz, ${ }^{24}$ Dorner ${ }^{25}$ ). This bradycardia disappears after the injection of atropine just as does the ordinary bradycardia seen during convalescence in other acute diseases. If the vasomotor paresis was the chief factor, we should furthermore find the veins of the neck empty; on the contrary, we find them distended and pulsating as the result of the simultaneous contractions of the auricle and the ventricle.

Although I am unable to prove that the vasomotor paresis, vagal paresis or the like are not present, I think that 1 am justified in declaring that the myocarditis, and especially the early disturbances of the rhythm explain the clinical findings during the acute stage of diphtheria.

23 Krankh. d. Herzens, etc., 1909.

24 Jahrb. f. Kinderh., 1897, 45, p. 89.

w Studien 3, Path. 1. Behandl. d. Diph., 1918. 
With regard to the extrasystoles, I think it is very doubtful that they are the result of an active myocarditis. The usual time for their appearance coincides with the time when the blood pressure has reached its normal height. I would rather believe that they were a symptom of convalescence and were caused by resorptive or regenerative processes (fibrous or myogenic), which might either give rise to new impulses or to an exaggerated sensibility of the rissues.

It is certain that the patients, when the extrasystoles have again disappeared, either are cured or have reached a stage in which no further progress can be expected even by prolonged staying in bed.

The extrasystoles cannot be considered as a form of diphtheric paralysis as several authors (Schmaltz, ${ }^{28}$ Romberg, ${ }^{27}$ Mackenzie, ${ }^{17}$ Parkinson, Gosse and Gunson ${ }^{28}$ ) have found them during convalescence from other acute diseases (scarlet fever, typhoid fever, rheumatic fever). I have myself found them during convalescence from scarlet fever. That the extrasystole in some cases was the first, in a few even the sole, symptom of disease of the heart, can be explained by the fact that the acute lesion of the muscle was so slight that it did not give any available symptom in the acute stage, although the regenerative (resorptive) changes later gave rise to the heterogenic contractions.

\section{REEXAMINATIONS}

Mackenzie ${ }^{17}$ says that the damage of the myocardium in acute diseases often is not stationary but progressive. The time that passes before the recognition of the cardiac impairment depends on the extent of the damage and the rapidity of its progress, and the amount of effort to which the person is subjected.

As I have showed, acute myocarditis is common in patients suffering from grave diphtheria. It is now a question of the greatest importance, whether this myocarditis disappears without leaving any available clinical symptom or whether it may be considered as the origin of chronic disease of the heart, which sooner or later may result in heart failure.

Several authors have said that the myocarditis following diphtheria gives rise to chronic diseases of the heart (Baginsky, ${ }^{29}$ Romberg, ${ }^{23}$ Grancher, Boulloche and Babonneix ${ }^{30}$ ). Experimentally, Mollard and Regaud have proved this.

2* München. med. Wchnschr,, 1904, 41, p. 1419.

$n$ Deutsch. Arch. f. klin. Med., 1891, 48, p. 369; 1892, 49, p. 413.

28 Quarterly Jour. Med., 1920, 13, p. 363.

20 Diphtherie u. diph. Croup, 1913.

so Diphthérie, Nouv. Trait d. med. 
Schmaltz ${ }^{31}$ is the only one who has followed a greater number of patients methodically a year or more after they had diphtheria, and he found that 1 year after recovery $50 \%$ of 56 patients, 2 years after recovery $42.5 \%$ of 41 patients and 3 years after recovery $42 \%$ of 19 patients showed signs of functional inefficiency of the mitral valve. He thinks the systolic murmur is of special value for the diagnosis of the myocardial disease. Förster ${ }^{32}$ has seen such patients develop grave heart failure 1 to 4 years after their discharge.

With the object of throwing more light on this question I have tried to examine, 2 years or more after their discharge, those of my patients who had shown signs of myocarditis. When discharged 30 $(34 \%)$ of the 89 patients with myocarditis showed no abnormal heart sign; in the rest $66 \%$ either murmurs or dilatation was found.

In determining, at the reexamination, whether the patient had a normal heart I have taken into consideration the subjective symptoms of exhaustion as well as the physical signs. With regard to the former I have considered whether the patient easily tired, shortness of breath and throbbing of the heart in running or walking fast. These symptoms were often combined with pains in the precordium. As all patients who were reexamined were children-between 5 and 15 years of age-I think that these symptoms of distress must signify an impairment of the heart, especially when they occur in formerly healthy children. The parents also often told me that their children were quite changed after their attack of diphtheria; they were irritable and sulky. Often they would not play with other children as before and when allowed to choose they preferred quiet games.

As to the abnormal physical symptoms, the most interesting is the persistence of a systolic murmur or a dilatation. These murmurs cannot be accidental as they appeared in formerly normal hearts during an acute infection.

Of the 89 patients, 66 came to be reexamined; none of them had had an acute infection after discharge. In 46 were found symptoms like those in cases of mitral regurgitation. Most conspicuous was the systolic murmur, which might be soft but could be very harsh. Only in one case was a disturbed rhythm found (occasionally an extrasystole). Dilatation was often present. This mitral regurgitation must be the result not of a valvulitis, but of a progressive lesion of the myo-

\footnotetext{
11 Festschr. 3. Feier d. 50 jähr Besteh. d. Stadtkrankheit. 3 Dresden-Friederichstadt, 1899 , p. 157

32 Deutsch. Arch. f. klin. Med., 1906, 85, p. 11.
} 
cardium which has given rise to a more or less pronounced impairment of the functional efficiency of the heart muscle.

In 4 of these cases there had been only a doubtful acute myocarditis during the diphtheria but on reexamination 2 years later, a pronounced impairment of the heart was found.

The question now is what the future will be for these patients. I am afraid that the more or less strenuous struggle for life with new infections, hard work and in the female, last but not least, pregnancy (delivery) will prove to be too much for the from the beginning only slightly impaired heart, so that years later heart failure will set in in many of these patients as a result of the long forgotten diphtheria in early childhood.

\section{SUM MARY}

Five hundred and sixty-eight patients with diphtheria have been examined for symptoms of cardiac impairment; 118 of them who suffered from grave diphtheria have been especially examined. Furthermore, 8 cases that were fatal in the acute stage, have been followed thoroughly.

Clinical signs of acute myocarditis were found in $17 \%$ of the 568 cases and in $75 \%$ of the cases of grave diphtheria.

Typical symptoms of myocarditis were present in all fatal cases in the acute stage.

During the course of the diphtheria two distinctly different types of disturbance of the rhythm appeared:

(a) An "early" type which on an average appeared on the 8th day of illness. It started as organic heartblock; in the course of a few hours to a few days the rhythm, as a rule, became very complex and varying, but still it formed a distinct, easily recognizable type (flutter?). This disturbance explains the pallor, the coolness of the skin and the frequent attacks of syncope. All these patients died of heart failure in the acute stage of the diphtheria.

(b) A "late" type which consisted of extrasystoles appearing on an average on the $33 \mathrm{rd}$ day of illness. Among the 563 cases that survived the acute stage extrasystoles were found in $14 \%$ and in $61 \%$ of the grave cases. Of the patients with symptoms of myocarditis $81 \%$ had extrasystoles during convalescence. None of these patients died of heart failure.

In 4 cases of type $a$, histologic examinations of the aterioventricular node and bundle showed that this system was attacked in the same degree as the myocardium surrounding it. 
Electrocardiograms taken during the 5th week of illness were normal in 31 cases with muscular mitral insufficiency and extrasystoles. These last were found in only 3 patients; the 2 patients had extrasystoles of auricular origin while the extrasystole in the $3 \mathrm{rd}$ case arose from the junctional tissues.

On reexamining the patients at least 2 years after their discharge signs of impairment of the heart were found in more than two thirds of those who came to be reexamined. In some cases, in which reexamination showed pronounced impairment, there had been during the stay at the hospital only doubtful signs of myocarditis. The diphtheria must, therefore, be considered an important cause of heart failure later in life. 